\title{
A força muscular de mulheres idosas decresce principalmente após oito semanas de interrupção de um programa de exercícios com pesos livres
}

\author{
Vagner Raso ${ }^{1}$, Sandra Marcela Mahecha Matsudo ${ }^{1}$ e Victor Keihan Rodrigues Matsudo ${ }^{1}$
}

\section{RESUMO}

Este estudo teve como objetivo verificar o efeito da interrupção de um programa de exercícios com pesos livres sobre a força muscular de mulheres idosas através do teste de uma repetição máxima (1-RM). Para tanto, a amostra foi constituída por oito mulheres idosas saudáveis ( $\bar{x}: 64,3 \pm$ 7,6 anos) que foram envolvidas, previamente à interrupção, em um programa de exercícios com pesos livres durante 12 semanas, três vezes por semana, três séries de 10 repetições a 50\% 1-RM para seis tipos de exercícios para os membros superiores e inferiores. O teste 1-RM foi realizado imediatamente após a interrupção do programa e subseqüentemente a cada quatro semanas $\left(44^{\mathrm{a}}, 8^{\mathrm{a}}\right.$ e $12^{\mathrm{a}}$ semanas). Os valores demonstraram decréscimo estatisticamente significativo na força muscular de ambas as extremidades corporais, principalmente após a $8^{\mathrm{a}}$ semana de pausa. $\mathrm{O}$ decréscimo percentual na 12 a semana variou de $27,5 \%$ a $35,1 \%$ para a força muscular de membros inferiores e superiores, respectivamente; aconteceu, principalmente, nos membros superiores. Os membros inferiores preservaram mais a capacidade de manutenção da força muscular após a interrupção do treinamento $(22,9 \%$ a $71,9 \%)$ que os membros superiores $(-14,8 \%$ a $16,1 \%)$ quando os valores absolutos finais do período de destreinamento foram comparados com os valores iniciais do treinamento. Esses resultados permitem concluir que a interrupção de um programa

1. Centro de Estudos do Laboratório de Aptidão Física de São Caetano do Sul - Celafiscs

Recebido em: 24/7/01

Aceito em: 14/11/01

Endereço para correspondência:

Vagner Raso

Rua Marquês de Praia Grande, 540, ap. 32B

03129-110 - São Paulo, SP

E-mail: vagner.raso@bol.com.br de exercícios com pesos livres produz efeito negativo na força muscular de mulheres idosas, especialmente após a 8 a semana.

Palavras-chave: Envelhecimento. Destreinamento. Força muscular. Exercícios com pesos.

\section{ABSTRACT}

The muscle strength of elderly women decreases specially 8 weeks after interruption of a muscle strengthening training program

The purpose of this study was to verify the interruption effect of a muscle strengthening training program on muscle strength through one maximum repetition test in elderly women. Before interruption, eight healthy elderly women ( $x$ : $64.3 \pm 7.6 y r$.) were engaged in a muscle strengthening training program for 12 weeks, 3 times per week, 3 sets of 10 repetitions at $50 \%$ of one maximum repetition test (1-RM) in six exercises for both upper and lower limbs. The 1-RM test was performed immediately after the training period and subsequently every four weeks $\left(4^{\text {th }}, 8^{\text {th }}\right.$ and $12^{\text {th }}$ week). Values showed a statistically significant decrease on muscle strength of lower and upper limbs, specially after 8 weeks. In the $12^{\text {th }}$ week, lower and upper limb muscle strength decreased $27.5 \%$ to $35.1 \%$, respectively; howev$e r$, decrease was more remarkable in the upper limb. A greater capacity of muscle strength maintenance after training interruption was observed in the lower limb $(22.9 \%$ to $71.9 \%)$ than in the upper limb $(-14.8 \%$ to $16.1 \%)$ when the absolute values of the detraining period were compared to the values at the beginning of the training period. These data allowed the authors to conclude that the weight training program interruption leads to a negative impact on muscle strength of elderly women, specially after 8 weeks.

Key words: Aging. Detraining. Muscle strength and muscle strength training. 


\section{INTRODUÇÃO}

O nosso serviço previamente registrou que um adequado programa de exercícios com pesos livres produziu incremento significativo na força muscular de ambas as extremidades corporais em mulheres idosas $(135,2 \%$ e $66,8 \%$ para membros inferiores e superiores, respectivamente) $)^{1}$. Esses ganhos observados na força parecem ser devidos provável e principalmente às adaptações neurais ${ }^{2-4}$, mas podem ser atribuídos ao incremento no tamanho da fibra muscular $^{5-8}$. Independente do mecanismo, os exercícios com pesos melhoram significativamente $(\mathrm{p}<0,05)$ a velocidade para levantar-se de uma posição sentada $(8,9 \%)$ e para subir escada $(4,3 \%)^{9-11}$.

No entanto, as adaptações morfológicas e funcionais crônicas induzidas pela atividade física regular são reduzidas ou retornam à situação anterior ao treinamento quando o programa de exercícios é interrompido. Esse fenômeno tem sido denominado de "efeito da pausa de treinamento" ou simplesmente "destreinamento" e a magnitude da reversibilidade é proporcional ao período de ausência de estímu$\mathrm{lo}^{12}$. A restrição para realizar atividades físicas cotidianas (como, por exemplo, o repouso em cama), a imobilização e viagens espaciais ${ }^{13}$, infecções ${ }^{14}$ e o envelhecimento per $s e^{15}$ são algumas outras condições que deterioram a capacidade do sistema muscular em realizar eficientemente suas funções.

Vários estudos têm reportado que a força muscular produzida por ações musculares isométricas, concêntricas, excêntricas, isotônicas e isocinéticas decresce após a interrupção de um programa de treinamento, independente da faixa etária, sexo, nível de aptidão física e enfermidades associadas ${ }^{16-22}$. Staron et al..$^{23}$ submeteram um grupo de mulheres adultas jovens, previamente treinadas em exercícios com pesos durante 20 semanas, a um período de destreinamento de 30 a 32 semanas e verificaram decréscimo na força muscular dinâmica, porém a alteração não alcançou os níveis pré-treinamento. Outro estudo demonstrou que a interrupção de um programa provocou redução na área média das fibras musculares tipo I e II ${ }^{24}$. Da mesma forma, Colliander e Tesch ${ }^{16}$ observaram maior preservação do pico de torque concêntrico e excêntrico após a interrupção, quando o programa de exercícios com pesos foi constituído de ações concêntricas e excêntricas do que somente concêntricas.

Embora existam estudos que demonstrem os efeitos da interrupção subseqüente a um programa de exercícios com pesos em adultos de ambos os sexos, a quantidade de trabalhos que têm características semelhantes em populações idosas é extremamente baixa. Desse modo, este estudo teve como objetivo verificar o efeito de 12 semanas de inter- rupção de um programa de exercícios com pesos livres sobre a força muscular de mulheres idosas.

\section{CASUÍSTICA E MÉTODOS}

\section{Voluntárias}

A amostra foi constituída por oito mulheres idosas saudáveis na faixa etária de 56 a 81 anos de idade $(64,3 \pm 7,6$ anos), com $155,2 \pm 7,4 \mathrm{~cm}$ de altura e $67,5 \pm 9,9 \mathrm{~kg}$ de peso corporal. As voluntárias foram informadas sobre os objetivos deste estudo assim como sobre os benefícios e possíveis riscos à saúde. Também foram informadas de que a participação no estudo era voluntária e que poderiam desistir a qualquer momento. Após essas orientações, um termo de consentimento foi obtido de cada voluntária.

\section{Programa de treinamento}

A interrupção do treinamento foi precedida por um programa de exercícios com pesos livres realizado durante 12 semanas (três vezes por semana). As voluntárias engajaram-se em um programa de exercícios com pesos livres que consistia em realizar três séries de 10 repetições a $50 \%$ de uma repetição máxima (1-RM) para os exercícios supino reto, supino inclinado, flexão de cúbito, extensão de cúbito, leg press $45^{\circ}$ e agachamento, com pausa de dois minutos entre as séries e os exercícios. Antes e após cada sessão de treinamento, as voluntárias realizavam exercícios de alongamento para os membros superiores e os inferiores. As sessões de treinamento foram supervisionadas por professores de Educação Física. Foram constantemente medidas a pressão arterial e a freqüência cardíaca.

As voluntárias foram avisadas de que durante o período de interrupção não poderiam engajar-se em nenhum tipo de programa regular de atividade física. Mesmo assim, uma das voluntárias reportou aderir a um programa de hidroginástica de baixa intensidade (duas vezes por semana; 25 a 30 minutos por sessão) durante as quatro semanas finais do período de interrupção do programa. Todavia, esse fato parece não ter influenciado os resultados relativos, assim como absolutos, visto que essa voluntária experimentou fenômeno de magnitude similar ao das outras voluntárias, independente do exercício analisado. Portanto, é pouco provável que a hidroginástica tenha induzido qualquer efeito sobre a estrutura e a função musculares da referida voluntária.

Por outro lado, a reanálise dos dados feita sob a estratégia de exclusão da referida voluntária poderia induzir erro por falso-negativo, independente do alfa empregado - sendo importante referenciar o valor conservador adotado no presente estudo. Nesse caso, é provável que todas as alterações resultantes da interrupção que apresentassem valores inferiores a $14,3 \%$ fossem ocultadas e as demais tivessem magnitude diminuída. Portanto, o poder estatístico 
"encobriria" a plausibilidade biológica - por razões de cálculo amostral. Visto que os resultados da voluntária não representaram uma forma outlier e não alteraram a plausibilidade biológica do fenômeno (como discutido acima) fato este corroborado pela característica gaussiana da magnitude do evento entre as voluntárias, em que a amplitude do coeficiente de variação foi de $8,0 \%$ a $29,8 \%$ - foi, portanto, possível incluir a referida voluntária na análise.

\section{Teste de uma repetição máxima (1-RM)}

O teste 1-RM foi feito subseqüentemente em períodos de quatro semanas imediatamente após o final do programa de exercícios (pós-programa) e na 4a ${ }^{a}, 8^{\mathrm{a}}$ e $12^{\mathrm{a}}$ semanas de interrupção (quatro avaliações). $\mathrm{O}$ procedimento do teste foi realizar exercícios de alongamento para os grupamentos musculares específicos e, imediatamente após, uma série de dez repetições em cada exercício sem sobrecarga, a não ser a da própria barra, que era de 7,5kg para os exercícios supino reto e inclinado, de $5 \mathrm{~kg}$ para o exercício flexão de cúbito e de $1 \mathrm{~kg}$ para o extensão de cúbito. Os valores anteriores do teste 1-RM foram utilizados como parâmetro para o incremento da sobrecarga. Em seguida, o teste foi iniciado aumentando gradativamente a sobrecarga (aumento nunca superior a $10 \%$ ) até a voluntária conseguir realizar uma repetição com o máximo peso possível. Foi respeitado um período mínimo de dois minutos de recuperação entre as tentativas, sendo que o número de tentativas para alcançar 1-RM não ultrapassou três; a seguinte ordem de exercícios foi adotada: supino reto, leg press $45^{\circ}$, supino inclinado, agachamento, flexão e extensão de cúbito. O propósito da seqüência dos exercícios e das voluntárias realizarem o teste em duplas, proporcionando período de repouso mais prolongado, foi evitar a fadiga muscular.

Foi recomendado às voluntárias inspirarem antes de realizar o movimento, expirarem durante a fase positiva do movimento e novamente inspirarem quando o peso retornasse à posição inicial, para evitar a manobra de Valsalva ${ }^{25}$.

\section{Análise estatística}

A estatística descritiva foi utilizada por meio das medidas de tendência central (média aritmética) e de dispersão (desvio padrão). A análise de variância para medidas repetidas (1a avaliação x 2 a avaliação x 3 a avaliação x 4a avaliação) foi empregada para verificar a significância estatística dos resultados nos distintos períodos das avaliações e o post-hoc Tukey foi utilizado para localizar as possíveis diferenças. Também foi calculado o delta percentual, que foi determinado no período pós-programa e ao final das 12 semanas de pausa. O intervalo de confiança (IC 95\%) representa a amplitude numérica em que é provável os resultados ocorrerem repetindo o experimento e mantendo as mesmas condições. O nível de significância adotado foi de $\mathrm{p}<0,05$. O software Graph Pad Instat (versão 2.0) foi usado para os cálculos.

\section{RESULTADOS}

Como descrito anteriormente, o período de destreinamento foi precedido por um programa de exercícios com pesos livres com duração similar à da interrupção (12 semanas). Maiores detalhes sobre o programa foram publicados em trabalho anterior ${ }^{1}$.

$\mathrm{Na}$ tabela 1 podem ser observados os resultados antes do início do programa (PRÉ) e após 12 semanas (PÓS) do programa de exercícios com pesos livres e a variação percentual alcançada após o treinamento $(\Delta \%)$. Foi verificado incremento percentual na força muscular predita através do aumento da sobrecarga utilizada para executar o teste 1RM, que variou de $25,6 \%$ (flexão de cúbito) a 66,8\% (supino inclinado) para os membros superiores. Os membros inferiores apresentaram maior incremento na capacidade de gerar força muscular $(135,2 \%$ e $69,7 \%$ para o agachamento e leg press $45^{\circ}$, respectivamente). Os pequenos grupamentos musculares (estimados através dos exercícios flexão e extensão de cúbito) demonstraram menor incremento após o programa do que os grandes grupamentos musculares (supino reto e inclinado).

Em relação ao período de interrupção do treinamento (tabelas 2 a 8), os resultados demonstraram decréscimo significativo na força muscular dos membros superiores e inferiores, especialmente após a 8a semana de interrupção. Nos valores absolutos do teste 1-RM que são apresentados neste estudo, não estão inclusos os pesos das barras, mas somente a sobrecarga adicional a elas.

As letras a, b, c, e d inseridas nas tabelas representam as diferenças estatisticamente significativas entre as avaliações. Como pode ser observado na tabela 2, houve redução estatisticamente significativa nos valores médios do

\section{TABELA 1}

Alteração na força muscular após 12 semanas de um programa de exercícios com pesos livres

\begin{tabular}{lrrr}
\hline & PRÉ (kg) & PÓs (kg) & \multicolumn{1}{c}{$\boldsymbol{\Delta} \%$} \\
& & & \\
Supino reto & $29,1 \pm 6,2$ & $46,0 \pm 6,4^{*}$ & 58,0 \\
Supino inclinado & $24,7 \pm 5,2$ & $41,2 \pm 5,4^{*}$ & 66,8 \\
Flexão de cúbito & $19,5 \pm 3,9$ & $24,5 \pm 3,3^{*}$ & 25,6 \\
Extensão de cúbito & $16,5 \pm 2,8$ & $23,3 \pm 1,9 *$ & 41,2 \\
Agachamento & $44,2 \pm 16,3$ & $104,0 \pm 25,6^{*}$ & 135,2 \\
Leg press 45 & $76,6 \pm 25,0$ & $130,0 \pm 29,7^{*}$ & 69,7
\end{tabular}

\footnotetext{
* Todos os valores do pós-programa (PÓS) são estatisticamente diferentes $(p<0,05)^{1}$.
} Os valores referem-se à média \pm desvio padrão e ao delta percentual $(\Delta \%)$. 
exercício supino reto $(30,0 \%)$ ao final do período de interrupção do programa de exercícios, com variação percentual entre as distintas avaliações de $-20,5 \%$ a $6,6 \%$. O decréscimo significativo iniciou-se na 4a semana após a pausa, mas a maior redução percentual ocorreu entre a $4^{\mathrm{a}} \mathrm{e}$ a $8^{\mathrm{a}}$ semanas $(20,5 \%)$. Na 12 a semana houve tendência não significativa de aumento de $6,6 \%$. O decréscimo percentual para a carga mínima e a máxima desempenhada foi de $40,0 \%$ e $31,0 \%$, respectivamente. A variação dos resultados durante o período de pausa foi de $-33,3 \%$ a $20,0 \%$ (carga mínima) e de $-20,0 \%$ a $0,0 \%$ (carga máxima).

A capacidade de produção de força muscular declina gradativa e significativamente com o decorrer do período de interrupção também para o exercício supino inclinado (tabela 3). Foi verificada alteração percentual média $(-30,3 \%)$ similar àquela experimentada no desempenho do exercício supino reto $(-30,0 \%)$. A variação percentual média entre o valor mínimo e o máximo após as 12 semanas de pausa foi de $-29,4 \%$ a $-40,0 \%$, respectivamente. Com exceção da alteração de $-13,3 \%$ observada na $8^{a}$ semana para o valor mínimo, os maiores decréscimos percentuais tanto do valor máximo como do resultado médio ocorreram nas primeiras quatro semanas $(28,0 \%$ para o valor máximo e $18,2 \%$ para o médio). Isso demonstra que esse período causou maior impacto sobre a perda da força muscular que os demais, especialmente naqueles indivíduos que tinham melhor performance no teste 1-RM.

Foi verificada redução estatisticamente significativa no exercício flexão de cúbito após o período de interrupção. A diminuição percentual média foi de $32,2 \%$ ao final das 12 semanas de interrupção do programa de exercícios. Os resultados relativos $(\%)$ entre as semanas variaram de

TABELA 2

Efeito da interrupção de um programa de exercícios com pesos livres nos valores (média \pm desvio padrão) do teste 1-RM $(\mathrm{kg})$ e $\Delta \%$ para o exercício supino reto $(n=8)$ imediatamente ao final (Pós) e após quatro, oito e doze semanas

\begin{tabular}{|c|c|c|c|c|c|}
\hline & $\begin{array}{l}\text { Pós } \\
\text { (a) }\end{array}$ & $\begin{array}{c}\text { 4ạ semana } \\
\text { (b) }\end{array}$ & $\begin{array}{c}\text { 8ạ semana } \\
\text { (c) }\end{array}$ & $\begin{array}{c}\text { 12ª semana } \\
\text { (d) }\end{array}$ & $\begin{array}{c}\text { PÓs - 12a } \\
\Delta \%\end{array}$ \\
\hline Mínimo & 40,0 & 30,0 & 20,0 & 24,0 & $-40,0$ \\
\hline Máximo & 58,0 & 50,0 & 40,0 & 40,0 & $-31,0$ \\
\hline Média $\pm D P$ & $44,7 \pm 6,5$ & $38,0 \pm 6,6^{a}$ & $30,2 \pm 6,0^{\mathrm{ab}}$ & $32,2 \pm 5,5^{\mathrm{ab}}$ & $-30,0$ \\
\hline IC 95\% & $39,3-50,2$ & $32,5-43,5$ & $25,2-35,3$ & $27,6-36,8$ & - \\
\hline $\begin{aligned} \Delta \% & \text { Mínimo } \\
& \text { Máximo } \\
& \text { Médio }\end{aligned}$ & & $\begin{array}{l}-25,0 \\
-13,7 \\
-17,3\end{array}$ & $\begin{array}{l}-33,3 \\
-20,0 \\
-20,5\end{array}$ & $\begin{array}{r}20,0 \\
0,0 \\
6,6\end{array}$ & \\
\hline
\end{tabular}

DP = desvio padrão; IC 95\% = valor inferior-valor superior; abcd $p<0,05$.

TABELA 3

Efeito da interrupção de um programa de exercícios com pesos livres nos valores (média \pm desvio padrão) do teste 1-RM $(\mathrm{kg})$ e $\Delta \%$ para o exercício supino inclinado $(n=8)$ imediatamente ao final (PÓS) e após quatro, oito e doze semanas

\begin{tabular}{|c|c|c|c|c|c|}
\hline & $\begin{array}{l}\text { Pós } \\
\text { (a) }\end{array}$ & $\begin{array}{c}\text { 4ạ semana } \\
\text { (b) }\end{array}$ & $\begin{array}{c}\text { 8a semana } \\
\text { (c) }\end{array}$ & $\begin{array}{c}\text { 12a semana } \\
\text { (d) }\end{array}$ & $\begin{array}{c}\text { Pós - 12a } \\
\Delta \%\end{array}$ \\
\hline Mínimo & 34,0 & 30,0 & 26,0 & 24,0 & $-29,4$ \\
\hline Máximo & 50,0 & 36,0 & 34,0 & 30,0 & $-40,0$ \\
\hline Média $\pm D P$ & $38,7 \pm 5,4$ & $33,7 \pm 1,6^{a}$ & $30,7 \pm 3,7^{a}$ & $28,7 \pm 2,4^{a b}$ & $-30,3$ \\
\hline IC 95\% & $34,2-43,3$ & $32,3-35,1$ & $27,7-33,8$ & $26,8-30,7$ & - \\
\hline $\begin{aligned} \Delta \% & \text { Mínimo } \\
& \text { Máximo } \\
& \text { Médio }\end{aligned}$ & & $\begin{array}{l}-11,7 \\
-28,0 \\
-18,2\end{array}$ & $\begin{array}{r}-13,3 \\
-5,5 \\
-8,9\end{array}$ & $\begin{array}{r}-7,6 \\
-11,7 \\
-6,5\end{array}$ & \\
\hline
\end{tabular}

DP = desvio padrão; IC 95\% = valor inferior-valor superior; abcd $\mathrm{p}<0,05$ 
$-37,5 \%$ na $8^{\text {a }}$ semana (valor mínimo) a $40,0 \%$ na $12^{a}$ semana (valor mínimo). As maiores perdas foram verificadas entre a 8 a e a $12^{\underline{a}}$ semanas no valor máximo $(25,0 \%)$, e no período entre a 4a e a $8^{\mathrm{a}}$ semanas, tanto para a variação percentual do valor mínimo $(37,5 \%)$ como para a do valor médio $(19,0 \%)$ (tabela 4). No entanto, a resposta à interrupção na $4^{\mathrm{a}}$ semana foi similar para os sujeitos que apresentavam pior ou melhor performance no 1-RM $(-20,0 \%)$.

No exercício extensão de cúbito foi observado decréscimo significativo na força muscular a partir da $8^{\underline{a}}$ semana de interrupção do programa de exercícios $(31,2 \%)$ quando comparado com os resultados executados na 4 a semana, alcançando ao final do período de interrupção a percentagem de $-35,1 \%$ (tabela 5).

Em relação a todos os exercícios direcionados para as extremidades superiores, os valores de execução do teste
1-RM no exercício extensão de cúbito demonstraram a maior alteração percentual $(-35,1 \%)$, em que a amplitude dos resultados para os exercícios foi de $-35,1 \%$ a $-30,0 \%$. Quando o valor máximo do exercício extensão de cúbito foi analisado, observou-se menor decréscimo percentual $(3,8 \%)$ após as 12 semanas de interrupção quando comparado com os demais exercícios. O maior decréscimo percentual foi observado independente do valor (mínimo, máximo ou médio) entre a 4 a e 8 a semanas, variando de $-11,5 \%$ a $-40,0 \%$. Ao contrário dos exercícios supino reto e inclinado, tanto o exercício de flexão como o de extensão de cúbito apresentaram decréscimo significativo somente na 8 a semana, sugerindo talvez que, embora estes exercícios tenham tido as maiores perdas absolutas quando comparados com os dois anteriores, esta região corporal parece ter maior capacidade para preservar a força muscular durante um período

\begin{tabular}{|c|c|c|c|c|c|}
\hline \multicolumn{6}{|c|}{$\begin{array}{c}\text { TABELA } 4 \\
\text { Efeito da interrupção de um programa de exercícios com pesos livres nos valores } \\
\text { (média } \pm \text { desvio padrão) do teste 1-RM }(\mathrm{kg}) \text { e } \Delta \% \text { para o exercício flexão de } \\
\text { cúbito }(\mathrm{n}=8) \text { imediatamente ao final }(\mathrm{PO} S) \text { e após quatro, oito e doze semanas }\end{array}$} \\
\hline & $\begin{array}{l}\text { PÓS } \\
\text { (a) }\end{array}$ & $\begin{array}{l}4^{a} \text { semana } \\
\text { (b) }\end{array}$ & $\begin{array}{l}8^{a} \text { semana } \\
\text { (c) }\end{array}$ & $\begin{array}{l}\text { 12a semana } \\
\text { (d) }\end{array}$ & $\begin{array}{c}\text { PÓs - 12a } \\
\Delta \%\end{array}$ \\
\hline Mínimo & 20,0 & 16,0 & 10,0 & 14,0 & $-30,0$ \\
\hline Máximo & 30,0 & 24,0 & 24,0 & 18,0 & $-40,0$ \\
\hline Média \pm DP & $23,2 \pm 2,8$ & $21,0 \pm 3,5$ & $17,0 \pm 4,5^{a}$ & $16,6 \pm 1,9^{\mathrm{ab}}$ & $-32,2$ \\
\hline IC 95\% & $20,9-25,6$ & $18,0-23,9$ & $13,2-20,8$ & $15,0-18,2$ & - \\
\hline $\begin{aligned} & \Delta \% \text { Mínimo } \\
& \text { Máximo } \\
& \text { Médio }\end{aligned}$ & & $\begin{array}{l}-20,0 \\
-20,0 \\
-14,2\end{array}$ & $\begin{array}{r}-37,5 \\
0,0 \\
-19,0\end{array}$ & $\begin{array}{r}40,0 \\
-25,0 \\
-2,3\end{array}$ & \\
\hline
\end{tabular}

TABELA 5

Efeito da intemupção de um programa de exercícios com pesos livres nos valores (média \pm desvio padrão) do teste 1-RM ( $\mathrm{kg})$ e $\Delta \%$ para o exercício extensão de cúbito $(n=8)$ imediatamente ao final (PÓs) e após quatro, oito e doze semanas

\begin{tabular}{lccccc}
\hline & $\begin{array}{c}\text { Pós } \\
\text { (a) }\end{array}$ & $\begin{array}{c}\text { 4a semana } \\
\text { (b) }\end{array}$ & $\begin{array}{c}\mathbf{8}^{\mathbf{a}} \mathbf{s e m a n a} \\
\text { (c) }\end{array}$ & $\begin{array}{c}\text { 12a semana } \\
\text { (d) }\end{array}$ & $\begin{array}{c}\text { Pós - 12a } \\
\mathbf{\Delta} \%\end{array}$ \\
Mínimo & 20,0 & 20,0 & 12,0 & 10,0 & $-50,0$ \\
Máximo & 26,0 & 26,0 & 23,0 & 25,0 & $-3,8$ \\
Média \pm DP & $23,4 \pm 1,9$ & $22,1 \pm 1,7$ & $15,2 \pm 3,4^{\text {ab }}$ & $15,1 \pm 4,6^{\text {ab }}$ & $-35,1$ \\
IC 95\% & $21,8-25,0$ & $20,7-23,6$ & $12,4-18,1$ & $11,3-18,9$ & - \\
$\Delta \%$ Mínimo & & 0,0 & $-40,0$ & $-16,6$ & \\
$\quad$ Máximo & & 0,0 & $-11,5$ & 8,6 & \\
Médio & & $-5,1$ & $-31,2$ & $-0,6$ & \\
\hline
\end{tabular}

DP = desvio padrão; IC 95\% = valor inferior-valor superior; abcd $p<0,05$ 
de tempo relativo (oito semanas); sendo assim, é necessário maior período total de interrupção para observar alterações significativas. Essa alusão é corroborada pelos valores de $\Delta \%$, enquanto na 4 a semana houve decréscimo significativo de $17,3 \%$ e de $18,2 \%$ nos valores de 1 -RM para os exercícios supino reto e supino inclinado, respectivamente. Para os exercícios flexão e extensão de cúbito, houve tendência não significativa de $-14,2 \%$ e $-5,1 \%$, respectivamente. Paradoxalmente, as respectivas situações na $8^{\mathrm{a}}$ semana foram de $-20,5 \%$ e $-8,9 \%$, e de $-19 \%$ e $-31,2 \%$ (todos estatisticamente significativos).

O exercício agachamento foi o único que não apresentou alteração estatisticamente significativa $(\mathrm{p}>0,05)$ ao final do período de interrupção (tabela 6). Os resultados demonstraram decréscimo percentual médio de 26,9\%. A variação percentual de diminuição entre os valores míni- mo e máximo foi de $18,9 \%$ e $26,6 \%$, respectivamente. Foi observada, na $8^{\text {a }}$ semana de interrupção, queda dramática no valor inferior do IC 95\% $(51,4)$ em relação à 4⿳亠丷厂 semana $(81,8)$, sugerindo que o decréscimo da força muscular neste período de tempo foi maior entre os sujeitos que desempenhavam o teste 1-RM com menor sobrecarga. As maiores alterações nos valores mínimo e máximo ocorreram na $4^{a}$ semana $(18,9 \%$ e $26,6 \%$, respectivamente), enquanto para o valor médio a magnitude de perda foi similar tanto na $4^{\mathrm{a}}$ $(11,9 \%)$ como na $8^{\text {a }}$ semana $(15,8 \%)$.

A tabela 7 apresenta os dados referentes ao exercício leg press $45^{\circ}$. Houve decréscimo médio de $27,5 \%$ após as 12 semanas de pausa. Assim como ocorreu com os exercícios supino reto e extensão de cúbito, a redução percentual do resultado mínimo para o leg press $45^{\circ}$ foi maior $(45,4 \%)$ do que para o valor máximo $(31,5 \%)$.

\begin{tabular}{|c|c|c|c|c|c|}
\hline \multicolumn{6}{|c|}{$\begin{array}{c}\text { TABELA } 6 \\
\text { Efeito da interrupção de um programa de exercícios com pesos livres nos valores } \\
\text { (média } \pm \text { desvio padrão) do teste } 1-R M(k g) \text { e } \Delta \% \text { para o exercício agachamento } \\
(n=5) \text { imediatamente ao final (Pós) e após quatro, oito e doze semanas }\end{array}$} \\
\hline & $\begin{array}{l}\text { PÓs } \\
\text { (a) }\end{array}$ & $\begin{array}{c}4^{a} \text { semana } \\
\text { (b) }\end{array}$ & $\begin{array}{c}\text { 8a semana } \\
\text { (c) }\end{array}$ & $\begin{array}{c}\text { 12a semana } \\
\text { (d) }\end{array}$ & $\begin{array}{c}\text { PÓs - 12a } \\
\Delta \%\end{array}$ \\
\hline Mínimo & 74,0 & 60,0 & 60,0 & 60,0 & $-18,9$ \\
\hline Máximo & 150,0 & 110,0 & 120,0 & 110,0 & $-26,6$ \\
\hline Média $\pm D P$ & $110,0 \pm 23,4$ & $98,0 \pm 13,0$ & $80,6 \pm 23,5$ & $76,0 \pm 20,7$ & $-26,9$ \\
\hline IC 95\% & $80,1-139,1$ & $81,8-114,2$ & $51,4-109,8$ & $50,2-101,7$ & - \\
\hline $\begin{array}{l}\Delta \% \text { Mínimo } \\
\text { Máximo } \\
\text { Médio }\end{array}$ & & $\begin{array}{l}-18,9 \\
-26,6 \\
-11,9\end{array}$ & $\begin{array}{r}0,0 \\
9,0 \\
-15,8\end{array}$ & $\begin{array}{r}0,0 \\
-8,3 \\
-1,4\end{array}$ & \\
\hline
\end{tabular}

TABELA 7

Efeito da intemupção de um programa de exercícios com pesos livres nos valores (média \pm desvio padrão) do teste 1-RM (kg) e $\Delta \%$ para o exercício leg press $45^{\circ}(n=7)$ imediatamente ao final (PÓs) e após quatro, oito e doze semanas

\begin{tabular}{|c|c|c|c|c|c|}
\hline & $\begin{array}{l}\text { Pós } \\
\text { (a) }\end{array}$ & $\begin{array}{c}\text { 4a semana } \\
\text { (b) }\end{array}$ & $\begin{array}{c}\text { 8a semana } \\
\text { (c) }\end{array}$ & $\begin{array}{c}\text { 12a semana } \\
\text { (d) }\end{array}$ & $\begin{array}{c}\text { PÓs - 12a } \\
\Delta \%\end{array}$ \\
\hline Mínimo & 110,0 & 80,0 & 80,0 & 60,0 & $-45,4$ \\
\hline Máximo & 190,0 & 130,0 & 140,0 & 130,0 & $-31,5$ \\
\hline Média $\pm D P$ & $140,0 \pm 42,0$ & $105,7 \pm 19,0^{a}$ & $106,6 \pm 20,4^{a}$ & $94,3 \pm 25,1^{a}$ & $-27,5$ \\
\hline IC 95\% & $101,1-178,9$ & $88,1-123,3$ & $87,7-125,4$ & $71,1-117,5$ & - \\
\hline $\begin{array}{c}\Delta \% \text { Mínimo } \\
\text { Máximo } \\
\text { Médio }\end{array}$ & & $\begin{array}{l}-27,2 \\
-31,5 \\
-19,2\end{array}$ & $\begin{array}{r}0,0 \\
7,6 \\
-1,7\end{array}$ & $\begin{array}{r}-25,0 \\
-7,1 \\
-8,7\end{array}$ & \\
\hline
\end{tabular}

DP = desvio padrão; IC 95\% = valor inferior-valor superior; abcd $p<0,05$ 
Ao contrário dos membros superiores (exceção de supino inclinado [4a semana, valor máximo: $-28,0 \%$; e valor médio: $-18,2 \%]$ ), em que a alteração percentual entre as avaliações freqüentemente foi maior após a $8^{\mathrm{a}}$ semana, nos membros inferiores, com exceção do agachamento ( $8^{\underline{a}}$ semana: $-15,8 \%$ ), o declínio foi maior nas quatro primeiras semanas de pausa.

Foram observadas várias características quando os valores absolutos finais do período de interrupção (PÓs) foram comparados com os valores iniciais do treinamento (PRÉ) (tabela 8). Os membros inferiores preservaram mais a capacidade de manutenção da força muscular após a interrupção do treinamento $(22,9 \%$ a $71,9 \%)$ que os membros superiores $(-14,8 \%$ a $16,1 \%)$.

\section{DISCUSSÃO}

As medidas foram divididas em níveis estrutural e funcional para tornar mais clara e específica a compreensão dos fenômenos observados no presente estudo. No plano transversal, os exercícios supino reto, supino inclinado, flexão e extensão de cúbito foram considerados como membros superiores enquanto o agachamento e o leg press $45^{\circ}$ como membros inferiores. Ao contrário do que ocorreu durante o programa de treinamento, a força muscular dos membros superiores apresentou maior declínio induzido pela interrupção do que a de membros inferiores. Provavelmente, esses resultados sugerem que o incremento da força muscular dos membros inferiores alcançado com o programa de treinamento tenha aumentado a atividade física diária espontânea das voluntárias, fato que poderia parcialmente explicar a maior capacidade de retenção da força adquirida nessa região.

Os grupamentos musculares foram analisados de acordo com o volume de músculos envolvidos na ação de um determinado exercício, sendo divididos em pequenos (flexão

\section{TABELA 8}

\section{Valores absolutos (kg) do teste 1-RM no início do programa de exercícios com pesos livres (PRÉ) e após 12 semanas de interrupção (PÓS) ${ }^{1}$}

\begin{tabular}{lrrr}
\hline & \multicolumn{1}{c}{ PRÉ } & PÓs & \multicolumn{1}{c}{$\boldsymbol{\Delta} \mathbf{\%}^{\mathbf{2}}$} \\
& & & \\
Supino reto & $29,1 \pm 6,2$ & $32,2 \pm 5,5$ & 10,6 \\
Supino inclinado & $24,7 \pm 5,2$ & $28,7 \pm 2,3$ & 16,1 \\
Flexão de cúbito & $19,5 \pm 3,9$ & $16,6 \pm 2,3$ & $-14,8$ \\
Extensão de cúbito & $16,5 \pm 2,8$ & $15,1 \pm 4,5$ & $-8,5$ \\
Agachamento & $44,2 \pm 16,3$ & $76,0 \pm 20,7$ & 71,9 \\
Leg press 45 & $76,6 \pm 25,0$ & $94,2 \pm 25,0$ & 22,9 \\
\hline
\end{tabular}

${ }_{1}^{1}$ Os valores referem-se à média \pm desvio padrão; ${ }^{2}$ Representa a variação percentual entre os dois períodos (PRÉ e PÓS). e extensão de cúbito) e grandes grupamentos musculares (supino reto e inclinado) ${ }^{26,27}$. Tanto os pequenos como os grandes grupamentos musculares diminuíram significativamente a força muscular após o período de 12 semanas de interrupção. No entanto, houve tendência de maior decréscimo nos pequenos grupamentos musculares $(35,1 \%$ : extensão de cúbito) que nos grandes $(30,0 \%$ : supino reto). Quando comparados com os valores do pré-treinamento, os pequenos grupamentos musculares apresentaram maior decréscimo percentual $(14,8 \%$ e $8,5 \%$, flexão e extensão de cúbito, respectivamente) do que os grandes grupamentos musculares, que diminuíram, mas ainda preservaram $10,6 \%$ da capacidade adquirida com o treinamento para o supino reto e $16,1 \%$ para o supino inclinado (tabela 8 ).

Embora os exercícios de flexão e extensão de cúbito utilizados neste estudo se constituam tanto de ações concêntricas como excêntricas e, portanto, se caracterizando como isotônicos, foi adotado para fins de análise o termo concêntrico para o exercício flexão de cúbito (por tratar-se de um exercício que tem o objetivo de estimular os músculos da face anterior do braço, que por sua vez apresenta como músculos motores primários o bíceps braquial e o braquial. Como sinergistas, o braquiorradial, extensores radiais longo e curto do carpo, pronador redondo, flexor radial do carpo, palmar longo e flexor ulnar do carpo, todos com ação concêntrica no momento positivo da execução). $\mathrm{O}$ termo excêntrico foi empregado para o exercício extensão de cúbito (pelo fato de ter o propósito de estimular os músculos da face posterior do braço, sendo músculos motores primários o tríceps braquial e o ancôneo, ambos com ação excêntrica no momento positivo da execução) ${ }^{26-28}$. Houve maior tendência de preservação da força muscular na ação concêntrica $(32,2 \%)$ do que no movimento excêntrico $(35,1 \%)$, mesmo que a magnitude da diferença tenha sido discreta $(2,9 \%)$. A característica de perda diferencial também foi observada considerando os valores iniciais do treinamento (pré-programa) com os valores finais do período de interrupção (tabela 8). O exercício flexão de cúbito perdeu $74,1 \%(-14,8 \%)$ da força muscular quando comparado com exercício extensão de cúbito $(-8,5 \%)$.

Os dados deste estudo não fornecem evidências precisas de que o decréscimo da força muscular após o período de interrupção tenha sido maior nas voluntárias que apresentavam piores ou melhores resultados na execução do teste 1-RM. Mas essa alusão pode ser sugerida através da variação percentual que ocorreu entre os valores mínimos e máximos após as 12 semanas de interrupção. Houve maior decréscimo percentual no valor mínimo nos exercícios supino reto, extensão de cúbito e leg press $45^{\circ}$, enquanto para os exercícios supino inclinado, flexão de cúbito e agachamento, a maior redução foi no valor máximo. O decrésci- 
mo percentual da força muscular induzido pela interrupção foi menor do que o ganho adquirido em resposta ao programa de exercícios com pesos de mesma duração ${ }^{1}$, sugerindo que provavelmente sejam necessárias mais de 12 semanas de interrupção para que os valores alcançados com o treinamento retornem à situação pré-treinamento.

Todavia, alguns estudos têm demonstrado que a força muscular pode ser preservada de cinco a até 31 semanas após o programa de exercícios ter sido interrompido ${ }^{29-32}$. Recentemente, Lemmer et al. ${ }^{32}$ observaram as repercussões provocadas na força muscular por um programa de exercícios com pesos de nove semanas, assim como pela sua subseqüente interrupção durante 31 semanas, de acordo com a faixa etária e sexo. Ao contrário do presente estudo, os autores demonstraram que a força muscular foi preservada mesmo após 12 semanas de interrupção do programa e que os valores eram ainda significativamente superiores aos encontrados antes do início do treinamento após 31 semanas de interrupção, mas foram estatisticamente inferiores quando comparados com os valores imediatamente após o treinamento e após 12 semanas de interrupção, independente da idade e sexo, com exceção das mulheres jovens. A magnitude de perda durante as 31 semanas de interrupção foi maior para os sujeitos mais velhos, embora não tenha havido nenhuma interação entre sexo e tempo. O decréscimo desigual na força muscular entre os sujeitos jovens e idosos no período de 12 a 31 semanas sugeriu que possa existir maior declínio no tamanho da fibra muscular e maior perda da eficiência do recrutamento das unidades motoras nos sujeitos idosos como efeitos induzidos pela interrupção do programa.

Ivey et al. ${ }^{33}$ registraram os dados referentes à máxima produção de força por unidade de massa muscular, referida como qualidade muscular (QM), obtidos nos mesmos sujeitos e durante o mesmo protocolo empregado por Lemmer et $a l^{32}$. Nesse estudo, a QM foi obtida por meio da performance no teste 1-RM (extensão de joelho [perna dominante]) dividida pelo volume muscular da perna dominante (obtido através de imagem de ressonância magnética) e expressa em $\mathrm{kg} / \mathrm{cm}^{3}$. Houve incremento estatisticamente significativo na força e volume muscular $(\mathrm{p}<0,05)$ assim como na QM ( $\mathrm{p}<0,01)$, independente da idade e sexo após o período de nove semanas de treinamento. A QM permaneceu acima dos valores anteriores ao treinamento, mesmo após 31 semanas de interrupção do programa para todos os grupos (masculino [jovem e idoso] e feminino [jovem e idoso]), com exceção do grupo de mulheres idosas. A preservação da QM durante o período de cessação foi atribuída ao menor declínio da força quando comparada com a perda de massa muscular. No entanto, Dutta $^{34}$ comenta que os dados do estudo de Ivey et al..$^{33}$ devem ser considerados com cautela, visto ser provável que indivíduos idosos tenham maior componente não contrátil na área de secção transversa muscular comparados com indivíduos jovens e, portanto, seria importante diferenciar os componentes contráteis dos não contráteis para determinar os efeitos do treinamento sobre a QM assim como sobre a reversibilidade dos efeitos.

Os achados de Taaffe e Marcus ${ }^{31}$ respaldam a sugestão do decréscimo do tamanho da fibra muscular proposta por Lemmer et al. ${ }^{32}$. Os autores observaram os efeitos da interrupção e subseqüiente retreinamento na morfologia e função musculares de 11 homens na faixa etária de 65 a 77 anos. Os sujeitos foram previamente submetidos a um programa de exercícios com pesos constituído de três séries de oito repetições a 75\% 1-RM para 10 exercícios, realizados três vezes por semana, durante 44 semanas. Após 12 semanas de destreinamento foi verificada redução de 29,9 $\pm 5,2 \%$ na força muscular quando comparada com os valores iniciais de ganho de força. A área de secção transversa, tanto das fibras tipo I como II, retornaram aos valores prétreinamento. No entanto, quando os indivíduos foram submetidos ao retreinamento, um curto período de tempo demonstrou ser suficiente para promover ganhos na força similares aos alcançados durante o treinamento. De modo oposto, as modestas melhoras na área de secção transversa induzidas pelo retreinamento sugerem que grande parte da reaquisição da força perdida com o destreinamento reflita adaptação neural mais do que hipertrofia.

Connelly e Vandervoort ${ }^{21}$ analisaram os efeitos de longo prazo da interrupção em 10 mulheres (idade média de 82,8 anos) residentes em asilo, após participarem de um programa de exercícios com pesos. A força muscular do quadríceps declinou 68,3\% ( $p<0,05)$ em relação aos valores do treinamento; houve decréscimo estatisticamente significativo na força isométrica de $24,4 \%\left(20^{\circ}\right), 28,7 \%\left(60^{\circ}\right)$ e $29,8 \%\left(90^{\circ}\right)$ para a flexão de joelho. A velocidade máxima e selecionada de caminhada, assim como o tempo para se levantar e correr, reduziu-se significativamente em $28,6 \%$ e $19,5 \%$, e não significativamente em $54,1 \%$, respectivamente. Quando os valores da interrupção foram comparados com os do pré-treinamento, o decréscimo na força isométrica variou de $0 \%$ a $14,3 \%$, enquanto na mobilidade funcional o intervalo foi de $16,5 \%$ a $20,7 \%$; para a força dinâmica o declínio foi de $48,9 \%$.

$\mathrm{O}$ fenômeno observado no presente estudo é similar àquele encontrado por outros autores e parece ser independente da presença de condições crônicas, embora a magnitude e as características da reta possam ser diferentes. Häkkinen et al..$^{22}$ observaram decréscimo significativo $(\mathrm{p}<0,001)$ na força muscular isométrica e dinâmica em indivíduos portadores de artrite reumatóide, sem alteração na forma da 
curva força-tempo. Os valores absolutos alcançados por essa população no teste após a interrupção do treinamento foram muito menores do que os de indivíduos saudáveis ajustados pela idade e sexo.

Portanto, os resultados deste estudo suportam a hipótese de que a interrupção de um programa de exercícios com pesos produz efeitos negativos sobre a força muscular. Os dados demonstraram decréscimo significativo na capacidade de produção de força muscular dos membros inferiores e superiores, especialmente após a $8^{\mathrm{a}}$ semana de interrupção. Essa redução variou entre $-27,5 \%$ para os membros inferiores e $-35,1 \%$ para os superiores, e ocorreu principalmente para os membros superiores. Os sujeitos diminuíram em média sua capacidade de produzir força muscular para cada dia de interrupção das sessões do programa em $0,76 \%$ e $0,97 \%$ para membros inferiores e superiores, respectivamente. Os membros inferiores preservaram mais a capacidade de manutenção da força muscular após a interrupção do treinamento $(22,9 \%$ a $71,9 \%)$ que os membros superiores $(-14,8 \%$ a $16,1 \%)$, mesmo quando os valores absolutos finais do período de interrupção foram com-

\section{REFERÊNCIAS}

1. Raso V, Andrade EL, Matsudo SMM, Matsudo VKR. Exercícios com pesos para mulheres idosas. Rev Bras Ativ Física Saúde 1997;2:17-26.

2. Moritani T, de Vries HA. Neural factors versus hypertrophy in the time course of muscle strength gain. Am J Phys Med 1979;58:115-30.

3. Moritani T, de Vries HA. Potential for gross muscle hypertrophy in older men. J Gerontol 1980;35:672-82.

4. Raso V, Matsudo SMM, Matsudo VKR. Influencia del aumento de la fuerza muscular sobre la circunferencia de brazo de mujeres ancianas. Gerontologia 2001 (em revisão).

5. Frontera WR, Meredith CN, O'Reilly KP, Knuttgen HG, Evans WJ. Strength conditioning in older men: skeletal muscle hypertrophy and improved function. J Appl Physiol 1988;64:1038-44.

6. Fiatarone MA, Marks EC, Ryan ND, Meredith CN, Lipsitz LA, Evans WJ. High intensity strength training in nonagenarians. Effects on skeletal muscle. JAMA 1990;263:3029-34.

7. Charette SL, Mcevoy L, Pyka G, Snow-Harter C, Guido D. Muscle hypertrophy response to resistance training in older women. J Appl Physiol 1991;70:1912-6.

8. Phillips W, Hazeldene R. Strength and muscle mass changes in elderly men following maximal isokinetic training. Gerontology 1996;42:11420.

9. Raso V, Casagrande M, Brito CA, Andrade EL, Matsudo SMM. Impacto do treinamento de força muscular de intensidade moderada sobre a performance nas atividades da vida diária em mulheres acima de 50 anos - Projeto Americana. Anais do XXI Simpósio Internacional de Ciências do Esporte, São Paulo 1998:87.

10. Raso V, Andrade EL, Matsudo SMM, Matsudo VKR. Aerobic or muscle strength exercise improve daily active living performance in elderly women? Med Sci Sports Exerc (Suppl) 1999;31:S1766.

11. Raso V, Matsudo SMM, Matsudo VKR. Exercício aeróbico ou com pesos melhora a performance nas atividades da vida diária de mulheres idosas? Gerontologia 2001 (em revisão). parados com os valores iniciais do treinamento. Portanto, esses resultados corroboram que a continuidade do programa de exercícios com pesos com intensidade suficiente para minimizar/prevenir a redução da força muscular é também necessária para pessoas idosas. Todavia, fornecem evidências de que, apesar do claro impacto negativo, talvez seja provável que o indivíduo possa interromper o programa durante aproximadamente quatro a seis semanas, sem, neste período, induzir qualquer ou mínima repercussão sobre a capacidade muscular, visto que os efeitos negativos associados à interrupção ocorreram principalmente após oito semanas. Neste caso, evidentemente, não se podem considerar eventuais infecções oportunísticas e/ou agravo das complicações de determinadas doenças crônico-degenerativas comuns a essa população.

\section{AGRADECIMENTOS}

Os autores expressam os seus agradecimentos à Academia Modulus (unidade Americana) por subsidiar parcialmente a realização dos projetos que envolvem intervenção com programas de exercícios com pesos em pessoas idosas.

12. Kraemer WJ, Fleck SJ. Designing resistance training programs. $2^{\text {nd }}$ ed Human Kinetics, 1997.

13. Bloomfield AS, Coyle EF. Bed rest, detraining, and retention of training-induced adaptations. In: American College of Sports Medicine. ACSM's Resource manual for guidelines for exercise testing and prescription. $2^{\text {nd }}$ ed. USA: Lea and Febiger, 1993:115-28.

14. Friman G, Wesslén L. Infections and exercise in high-performance athletes. Immunol Cell Biol 2000;78:510-22.

15. Ross R, Heymsfield SB. Sarcopenia (muscle wasting) and aging: significance of exercise - Introduction to the symposium. Can J Appl Physiol 2001;26:76-7.

16. Colliander EB, Tesch PA. Effects of detraining following short term resistance training on eccentric and concentric muscle strength. Acta Physiol Scand 1992;144:23-9.

17. Houmard JA, Hortobágyi T, Johns RA, Bruno NJ, Nute CC, Shinebarger $\mathrm{MH}$, et al. Effect of short-term training cessation on performance measures in distance runners. Int J Sports Med 1992;13:572-6.

18. Madsen K, Pedersen PK, Djurhuus MS, Klitgaard NA. Effects of detraining on endurance capacity and metabolic changes during prolonged exhaustive exercise. J Appl Physiol 1993;75:1444-51.

19. Fleck SJ. Detraining: its effects on endurance and strength. J Strength Cond Res 1994:22-8.

20. McCoy M, Proietto J, Hargreaves M. Effect of detraining on GLUT-4 protein in human skeletal muscle. J Appl Physiol 1994;77:1532-6.

21. Connelly DM, Vandervoort AA. Effects of detraining on knee extension strength and functional mobility in a group of elderly women. J Orthop Sports Phys Ther 1997;26:340-6.

22. Häkkinen A, Malkia E, Häkkinen K, Jappinen I, Laitinen L, Hannonen P. Effects of detraining subsequent to strength training on neuromuscular function in patients with inflammatory arthritis. Br J Rheumatol 1997; 36:1075-81. 
23. Staron RS, Leonardi MJ, Karapondo DL, Malicky ES, Falkel JE, Hagerman FC, Hikida RS. Strength and skeletal muscle adaptations in heavyresistance-trained women after detraining and retraining. J Appl Physiol 1991;70:631-40

24. Häkkinen K, Alén M, Komi PV. Changes in isometric force and relaxation-time, electromyographic and muscle fibre characteristics of human skeletal muscle during strength training and detraining. Acta Physiol Scand 1985;125:573-85.

25. Feigenbaum MS, Pollock ML. Prescription of resistance training for health and disease. Med Sci Sports Exerc 1999;31:38-45.

26. Rasch PJ. Cinesiologia e anatomia aplicada. 7a ed. Rio de Janeiro: Guanabara Koogan, 1991.

27. Hall S. Biomecânica básica. Rio de Janeiro: Guanabara Koogan, 1993.

28. Kendall FP, McCreary EK. Músculos: provas e funções. 3a ed. São Paulo: Manole, 1990.

29. Lexell J, Downham DY, Larsson Y, Bruhn E, Morsing B. Heavy resistance training in older Scandinavian men and women: short- and long- term effects on arm and leg muscles. Scand J Med Sci Sports 1995;5: $329-41$.

30. Sforzo GA, McManis BG, Black D, Lunierwski D, Scriber KC. Resilience to exercise detraining in healthy older people. J Am Geriatr Soc 1995;43:209-15.

31. Taaffe DR, Marcus R. Dynamic muscle strength alterations to detraining and retraining in elderly men. Clin Physiol 1997;17:311-24.

32. Lemmer JT, Hurlbut DE, Martel GF, Tracy BL, Ivey FM, Metter EJ, et al. Age and gender responses to strength training and detraining. Med Sci Sports Exerc 2000;32:1505-12.

33. Ivey FM, Tracy BL, Lemmer JT, NessAiver M, Metter EJ, Fozard JL, et al. Effects of strength training and detraining on muscle quality: age and gender comparisons. J Gerontol Biol Sci 2000;55A:B152-7.

34. Dutta C. Commentary on effects of strength training and detraining on muscle quality: age and gender comparisons. J Gerontol Biol Sci 2000; 55A:B158-9. 